\title{
Technical Skill Assessment using Machine Learning and Artificial Intelligence Algorithm
}

\author{
Mohammad Suhaib Ahmed \\ Dept of Computer Engineering \\ DYPIEMR Akurdi, Pune \\ $(S P P U)$ Pune, India
}

Nikhunj Saraf

Dept of Computer Engineering

DYPIEMR Akurdi, Pune

(SPPU) Pune, India

\author{
Ajinkya Bhujbal \\ Dept of Computer Engineering \\ DYPIEMR Akurdi, Pune \\ $(S P P U)$ Pune, India
}

\author{
Rohan Atale \\ Dept of Computer Engineering \\ DYPIEMR Akurdi, Pune \\ $(S P P U)$ Pune, India
}

\begin{abstract}
This project is for every programmer whether they are absolute beginners or a pro at coding. There are a lot of websites that teach programming languages or provide you with the platform to solve programming language. But they often ignore the personality and skill level of the programmer. If you sign up for the first time you have to do all the basic stuff first in order to get to your level. We propose a system where we test the programmer first with basic to hard programming questions in the language of their choice and then software decides the skill level of the programmer. the course and the problem will be set according to the programmer skills and it will change accordingly with the growth.
\end{abstract}

Keywords - Machine Learning, Personalized Learning, Skill Assessment, Artificial Algorithm

\section{INTRODUCTION}

Education is moving far from ancient rows of scholars gazing at a similar textbook whereas a teacher lectures from the front of classroom. Today's school rooms aren't merely evolving to use additional technology and digital resources; they additionally finance in machine learning. (1) personalized learning refers to instruction during which the pace of learning and therefore the tutorial approach are optimized for the needs of each learner. (2) to induce a concept of what personalized learning is, try and imagine a room that doesn't have a "one size fits all" approach to education. The teacher doesn't lead all students through similar lessons. Instead, the teacher guides every student on a personalized journey. The what, when, wherever and the way of learning is ready-made to satisfy every student's strengths, skills, needs, and interests. personalized learning doesn't replace associate degree IEP, a 504 arrange or intervention programs. (3). during this project, we have a tendency to planned a system wherever the scholar will develop their ability victimization our personalized skill assessment tool and learn at their own pace.

There are a few key approaches toward personalized learning: (2)

a. Adaptive learning: technology who assign human or digital resources to learners to supported their distinctive wants.

b. Individualized learning: the pace of learning is adjusted to fulfill the needs of individual students. c. Differentiated learning: the approach to learning is adjusted to satisfy the wants of individual students.

d. Competence-based learning: learners advance through a learning pathway supported their ability to demonstrate competence, as well as the applying and creation of data along with side skills and tendencies

\section{LITERATURE SURVEY}

1. Baolin Yi; Dujuan Zhang (4), introduce a brand new approach to personalized Learning Model underneath informatization surroundings. thanks to the influence of scientific discipline and science, customized learning has bit by bit drawn the eye of the many researchers. the aim of this paper is to analysis a suitable customized learning technique for learners. during this paper, we have a tendency to perform a study by literature review from four aspects.

Firstly, we have a tendency to construct a learner model supported learner's learning vogue and psychological feature ability.

2. Alex Gulherms (5), describes the teacher and student relations. A process side of our trendy age is our tenacious belief in technology altogether walks of life, not least in education.

It might be argued that this infatuation with technology or 'techno-philia' in education has had a deep impact within the classroom changing the connection between teacher and student, moreover as between students; that's, these relations became progressively more I-it and then I-Thou primarily based as a result of the capability to make bonds, the amount of connectedness between teacher and students, and between students has either shrunken or become impaired by the increasing technologization of education.

3. Erkan Er (6), describes a way to characteristic Atrisk Students using Machine Learning Techniques, a model for predicting students' performance levels is projected that employs three machine learning algorithms: instance-based learning Classifier, Decision Tree and Naïve Thomas Bayes. additionally, three decision schemes were wont to mix the results of the 
machine learning techniques in several ways to analyze if higher classification performance might be achieved. The study conducted by Kotsiantis et al [1] is one of the initial studies that investigated the applying of machine learning techniques in distance learning for dropout prediction. during this study, time-invariant and time-varying information was enclosed and whole six machine learning techniques were utilized, that square measure call Trees, Neural Networks, Naïve Thomas Bayes algorithmic rule, Instance-Based Learning Algorithms, supplying Regression and Support Vector Machines.

The vital conclusion of this study is that Naïve Thomas Bayes algorithmic rule is extremely booming within the prediction of dropouts; it predicts with $83 \%$ accuracy.

4. In 2007, Formative Assessment for college students and lecturers State cooperative (FAST SCASS) (7) of the Council of Chief State Officers with national and international researchers in formative assessment known Five attributes of the formative assessment method from the literature. they're as follows:

I. Learning progressions ought to clearly articulate the sub-goals of the final learning goal.

II. Learning goals and criteria for fulfillment ought to be clearly known and communicated to students.

III. Students ought to be supplied with evidence-based feedback that's joined to the meant tutorial outcomes and criteria for fulfillment.

IV. Each self- and peer-assessment are necessary for providing students a chance to assume metacognitively concerning their learning.

V. A schoolroom culture within which lecturers and students are partners in learning ought to be established

John Hattie and Helen of Troy Timperley (10) describes in his Review of instructional analysis March 2007 of the facility of Feedback, Feedback is one among the foremost powerful influences on learning and action, however, this impact is often either positive or negative. Its power is often mentioned in articles concerning learning and teaching, however amazingly few recent studies have consistently investigated its which means. this text provides an abstract analysis offered back and reviews the proof associated with its impact on learning and action.

This proof shows that though. feedback is among the main influences, the kind of feedback and therefore the manner it's given are often differentially effective.
TABLE 2

Summary of effect sizes relating to feedback effects

\begin{tabular}{lcccc}
\hline Variable & $\begin{array}{c}\text { Number of } \\
\text { meta-analyses }\end{array}$ & $\begin{array}{c}\text { Number of } \\
\text { studies }\end{array}$ & $\begin{array}{c}\text { Number of } \\
\text { effects }\end{array}$ & $\begin{array}{c}\text { Effect } \\
\text { size }\end{array}$ \\
\hline Cues & 3 & 89 & 129 & 1.10 \\
Feedback & 74 & 4,157 & 5,755 & 0.95 \\
Reinforcement & 1 & 19 & 19 & 0.94 \\
Video or audio feedback & 1 & 91 & 715 & 0.64 \\
Computer-assisted & & & & \\
$\quad$ instructional feedback & 4 & 161 & 129 & 0.52 \\
Goals and feedback & 8 & 640 & 121 & 0.46 \\
Student evaluation feedback & 3 & 100 & 61 & 0.42 \\
Corrective feedback & 25 & 1,149 & 1,040 & 0.37 \\
Delayed versus immediate & 5 & 178 & 83 & 0.34 \\
Reward & 3 & 223 & 508 & 0.31 \\
Immediate versus delayed & 8 & 398 & 167 & 0.24 \\
Punishment & 1 & 89 & 210 & 0.20 \\
Praise & 11 & 388 & 4,410 & 0.14 \\
Programmed instruction & 1 & 40 & 23 & -0.04 \\
\hline
\end{tabular}

A model of feedback is then planned that identifies the actual properties and circumstances that build it effective, and a few usually thorny problems are mentioned, together with the temporal order of feedback and therefore the effects of positive and feedback. Finally, this analysis is employed to recommend ways in which within which feedback is often wont to enhance its effectiveness in lecture rooms.

6. Ms. Heritage describes what the skillful use of formative assessment would seem like. in What Do lecturers ought to recognize and Do? (11) She explained that Formative assessment if used effectively, will give lecturers and their students with the knowledge they have to maneuver to move forward. however, once over 100 years of exhortations and a big body of analysis on the subject, the concept that assessment and teaching are reciprocal activities remains not firmly placed within the observe of educators. Instead, assessment is usually viewed as one thing in competition with teaching, instead of as associate integral a part of teaching and learning. the educational progression ought to clearly articulate the sub-goals that represent progress toward the ultimate goal. Most state standards, by themselves, don't give a transparent progression for understanding wherever students are relative to desired goals.

In fact, several state standards don't even give a transparent image of what learning is anticipated. Developing learning progressions toward standards could be an essential component of formative assessment. Learning progressions give a large image of what's to be learned, and that they facilitate lecturers find students' current learning standing on the time on that student's square measure expected to progress.

James W. Pellegrino (12) who works in the Department of Psychology University of Illinois at Chicago, USA said in his paper on "The Challenge of Knowing What Student Know", Educators assess students to learn about what they know and can do, but assessments do not offer a direct pipeline into a student's mind. Assessing student knowledge and educational outcomes are not as straightforward as measuring height or weight; the attributes to be measured are mental representations and processes that are not outwardly visible. Thus, an assessment is a tool designed to observe 
students' behavior and produce data that can be used to draw reasonable inferences about what students know. Deciding what to assess and how to do so is not as simple as it might appear. Existing guidelines for assessment design emphasize that the process should begin with a statement of the purpose for the assessment and a definition of the content domain to be measured (AERA et al., 1999; Millman \& Greene, 1993).

In summary, Mislevy et al. provide us with a comprehensive model for educational assessment that clearly outstrips the current knowledge base. Furthermore, it may also contain a level of specificity that lacks conceptual and empirical justification. Much more evidence will be needed about the necessity, sufficiency, and utility of what they propose. Even so, the broader rationale for their system contains many important implications for assessment design, practice, and policy. We have a considerable distance to travel in meeting the scientific, practical and political challenges implied by evidence-centered design systems. Nonetheless, even small steps along the path of such systems will undoubtedly lead to significantly improved educational assessment.

8. Dylan Wiliam, (13) ETS wrote in his paper on Formative assessment: obtaining the main target right concerning the Writing in 1967, Michael Scriven steered 2 roles that analysis may play. On the one hand, " it's going to have a task within the on-going improvement of the curriculum" (Scriven, 1967 p.41) whereas in another role, " the analysis method could serve to alter directors to come to a decision whether or not the whole finished information, refined by use of the analysis method in its initial role, represents a sufficiently vital advance on the obtainable alternatives to justify the expense of adoption by a college system" (pp. 41-42). He then planned "to use the terms 'formative' and 'summative' analysis to qualify analysis in these roles."

What makes Associate in Nursing assessment formative, therefore, isn't the length of the circuit, nor wherever it takes place, nor UN agency carries it out, nor even UN agency responds. The crucial feature is that proof is elicited, taken in terms of learning wants, and accustomed to create changes to higher meet that learning wants.

\begin{tabular}{lll}
\hline Type & Focus & Length \\
\hline $\begin{array}{l}\text { Long-cycle } \\
\begin{array}{l}\text { Medium-cycle } \\
\text { Short-cycle }\end{array}\end{array}$ & $\begin{array}{l}\text { between instructional units } \\
\text { between lessons } \\
\text { within a single lesson }\end{array}$ & $\begin{array}{l}\text { four weeks to one year or more } \\
\text { one day to two weeks } \\
\text { five seconds to one hour }\end{array}$ \\
\hline & Table 1: Types of formative assessment
\end{tabular}

All the papers during this special issue highlight these aspects to a bigger or lesser extent. The analysis by Ruiz-Primo and Aracelli shows that the lecturers who most systematically elicit the proper forms of data (conceptual eliciting questions), who have ways in which of decoding the students' responses in terms of learning wants, and who will use this data to adapt their instruction, generate higher levels of student accomplishment.

Taken as a full, the papers during this issue build vital contributions to our understanding of however troublesome it's doubtless to be to enhance teachers' use of formative assessment methods. above all, they counsel that whereas the supply of high-quality tools is also a necessary condition, it's in no way a comfortable condition for the development of formative assessment observes. Tools for formative assessment can solely improve formative assessment practices if lecturers will integrate them into their regular schoolroom activities. In alternative words, the task of rising formative assessment is considerable, if not chiefly, regarding teacher skilled development.

9. Ibtehal Talal Nafea wrote a paper on topic Machine Learning in Education Technology wherever he talks regarding the role of metric capacity unit in Education by describing the however it will remodel learning to a good height. Customized and personalized learning (14) Machine learning is versatile enough to cater to all or any students despite their learning speeds.

By creating the use of algorithms that learn the way the scholar consumes data, machine learning permits the learner to maneuver ahead solely when they need actually grasped the previous content. This method ensures that no student is unnoticed or left behind. this is often true notwithstanding they're the sole one in school that has not however understood the content. The machine learning system conjointly permits lecturers to separately monitor student and facilitate them those areas wherever they're deficient.

This contrasts with the standard academic technique, that focuses on one-size-fits-all management wherever everybody in school is instructed constant manner. this kind of learning may be found within the EdTech and MagicBox learning systems

The future work on machine learning, particularly within the education context, shall witness the event of a lot of subtle AI tools. There square measure multiple prospects for coming up with complicated chatbots which will improve the sophistication of virtual assistants. This development shall foster a lot of human interactions which will replace emails and text messages. Already, plans square measure current for developing on-line virtual assistants named "Amy" or "Andrew" at x.ai to schedule conferences with each tutor and learners.

AI not to mention machine learning that includes deep learning and linguistic communication process is projected to travel level higher by incorporating a lot of subtle systems laced with capabilities to adapt, learn and predict systems with utmost autonomy. the longer term works on these systems shall incorporate a mixture of advanced algorithms and embedded huge knowledge sets.

10. S.B. Kotsiantis aforementioned in his Paper Use of Machine learning Techniques for academic proposes: a call network for prediction students' grades Use of machine learning techniques for academic proposes (or academic knowledge mining) is AN rising field aimed toward developing ways of exploring knowledge from machine academic settings and discovering purposeful patterns.

The hold on knowledge (virtual courses, e-learning $\log$ file, demographic and tutorial knowledge of scholars, 
admissions/registration information, then on) may be helpful for machine learning algorithms. As a lot of and a lot of students enter on-line learning environments, databases regarding student access and study patterns can grow.

Having data like check scores and alternative student data obtainable electronically will offer tutors with powerful tools for decision-making. we tend to hope that the data made by such call support tools may be usefully applied by tutors to attenuate the number of scholars United Nations agency square measure probable to fail by providing them with further teaching material or the other support. methods in usage of machine learning techniques for academic proposes embrace classification and regression algorithms, association rules, serial pattern analysis, likewise as cluster and internet mining (Baker and Yacef 2009).

In e-learning, classification has been used for grouping students as hint-driven or failure driven and finding students' common misconceptions (Yudelson et al. 2006); distinctive learners with very little motivation and finding remedial actions so as to lower drop-out rates (Cocea and Weibelzahl 2006) and for predicting course success (Hamalainen and Vinni2006) In this Paper we tend to learn however we will use totally different machine learning to our advantage within the planned project.

\section{PROPOSED APPROACH}

In proposed work, we will develop a system that would perfectly assess the students and provide them with the learning path personalized to them according to their goals and skills they currently have and what they want to achieve in the future. We would have to ask each user a certain type of question first to identify his profile and get a better understanding of its future goals.

After that pre-questionnaire, the student can give a generalized test to check his skills in various different fields in the computer and after the test, we will display his knowledge graph in each of these fields and what he should more focus on. The student can take more specialized tests in a particular subject to assess his hold on the subject. Our System would help student to provide a clear path by removing all the clutter. With the vast information we have on the internet we are more confused than educated.

Because we need an Algorithm to the classification of Student Skills based on his performance, we need to make use of Machine Learning Classification Algorithm Like Naïve Bayes and Logistic Regression

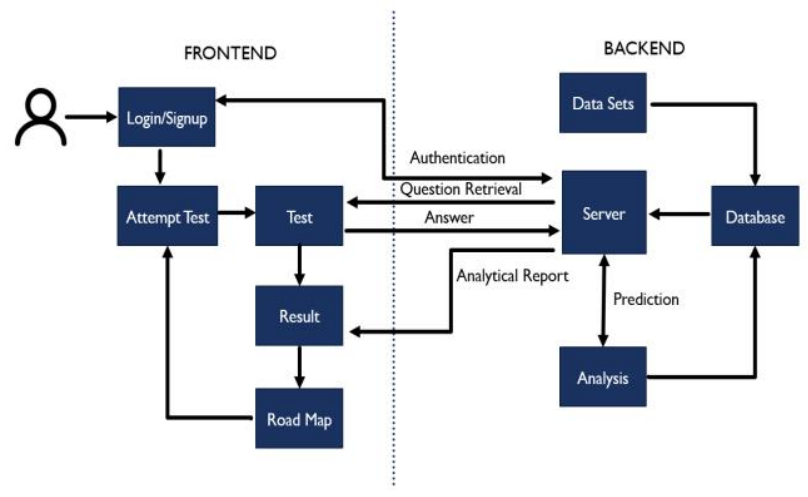

A. Algorithm Used

System Architecture

1. Naïve Bayes: It is a classification technique based on Bayes' Theorem with an assumption of independence among predictors. In simple terms, a Naive Bayes classifier assumes that the presence of a particular feature in a class is unrelated to the presence of any other feature.

2. Content-based (8) filtering methods are based on a description of the item and a profile of the user's preferences. These methods are best suited to situations where there is known data on an item (name, location, description, etc.), but not on the user. Content-based recommenders treat recommendation as a user-specific classification problem and learn a classifier for the user's likes and dislikes based on product features.

In this system, keywords are used to describe the items and a user profile is built to indicate the type of item this user likes. In other words, these algorithms try to recommend items that are similar to those that a user liked in the past, or is examining in the present. It does not rely on a user sign-in mechanism to generate this often temporary profile. In particular, various candidate items are compared with items previously rated by the user and the best-matching items are recommended. This approach has its roots in information retrieval and information filtering research.

\section{CONCLUSION AND FUTURE SCOPE}

Our System will be helpful for student who is preparing for College Placements because they can tune their skills and will get a brief idea about how to prepare for the company placement exam. It can also be helpful for the student of any age to hone their skills.

In Future Scope, we can add many more targeted features to help the student to prepare for the specific company and provide tutorial in the system so student doesn't need to roam on the internet to find notes.

\section{REFERENCES}

[1] https://www.thetechedvocate.org/8-ways-machine-learning-willimprove-education/

[2] https://medium.com/swlh/personalized-learning-throughartificial-intelligence-b01051d07494 
[3] https://www.understood.org/en/school-learning/partnering-withchilds-school/instructional-strategies/personalized-learning-whatyou-need-to-know.

[4] Baolin Yi ; Dujuan Zhang, "Research on Personalized Learning Model under Informatization Environment,".

[5] Alex Gulherms, "AI and education: the importance of teacher and student relations", March 2019 AI \& Society, Volume 34.s

[6] Erkan Er," Identifying At-Risk Students Using Machine Learning Techniques: A Case Study with IS 100". International Journal of Machine Learning and Computing, Vol. 2, No. 4, August 2012

[7] Ashford, S. J. (1986). Feedback-seeking in individual adaptation: A resource perspective. Academy of Management Journal, 29, 465-487.

[8] https://en.wikipedia.org/wiki/Recommender_system

[9] https://www.analyticsvidhya.com/blog/2017/09/naive-bayesexplained/
[10] John Hattie and Helen Timperley Review of Educational Research March 2007

[11] Margaret Heritage, "Formative Assessment: What Do Teachers Need to Know and Do?" OCTOBER 2007• Volume $89 \bullet$ Number

[12] James W. Pellegrino, "The Challenge of Knowing What Student Know" January 2005

[13] Dylan Wiliam, ETS "Formative assessment: getting the focus right about" 1967

[14] S.B. Kotsiantis, "Use of Machine learning Techniques for educational proposes: a decision support system for forecasting students grades"

[15] Ibtehal Talal Nafea "Machine Learning in Education Technology" July 3rd, 2017 July 3rd 2017 